\title{
The impact of non alcoholic fatty liver disease (NAFLD) on carotid artery intima-media thickness as a risk factor for atherosclerosis
}

\author{
Cristina Alina Silaghi ${ }^{1,2}$, Horațiu Silaghi ${ }^{3}$, Anca Elena Crăciun ${ }^{4,5}$, Andrada Oltean ${ }^{4}$, Daniel Tudor Cosma ${ }^{4}$, \\ Nicolae Hâncu ${ }^{5}$, Carmen Emanuela Georgescu²
}

${ }^{1}$ County Clinic Emergency Hospital, Cluj-Napoca, Romania; ${ }^{2}$ Endocrinology Department, ${ }^{3}$ Surgery V Department, ${ }^{4}$ Diabet, Nutrition and Metabolic Diseases Department,"luliu Hatieganu" University of Medicine and Pharmacy, Cluj-Napoca, Romania; ${ }^{5}$ Regina Maria Clinic, Cluj-Napoca, Romania.

\section{Introduction}

Nonalcoholic fatty liver disease (NAFLD) is a common and underdiagnosed chronic liver disease affecting up to one-third of the global population in Western countries. The prevalence of NAFLD is increased up to $70 \%$ to $90 \%$ among T2DM persons and makes it an important public health problem. NAFLD is considered a hepatic manifestation of metabolic syndrome and is closely associated with abdominal obesity, atherogenic dyslipidemia, and diabetes exposing subjects with NAFLD to an increased risk of developing cardiovascular disease. Carotid intima-media thickness (cIMT) is a reliable index of subclinical atherosclerosis and a mirror of atherosclerosis progression in NAFLD patients. Carotid IMT is higher for individuals with hepatosteatosis than for those with simple steatosis and the histological severity of NAFLD independently predicts CIMT $(1,2)$.

The aim of this study was to determine the prevalence of NAFLD in diabetes mellitus (DM) patients, to evaluate the impact of clinical parameters and indices of body composition on the relation between NAFLD and carotid intima-media thickness (cIMT), in a type 2 diabetes mellitus population (T2DM).

\section{Methods}

We retrospectively enrolled 120 T2DM outpatients who regularly attended a Consulting Clinic in Cluj. Clinical, anthropometric and biochemical parameters were measured. NAFLD was diagnosed using fatty liver index (FLI, 3), an algorithm based on body mass index (BMI), waist circumference, triglycerides and gammaglutamyl-transferase (GGT). Hepatic steatosis (HS) and cIMT were evaluated by ultrasonography (US). Body composition was assessed by bioelectric impedance (BIA, InBody 720 ) in all subjects.
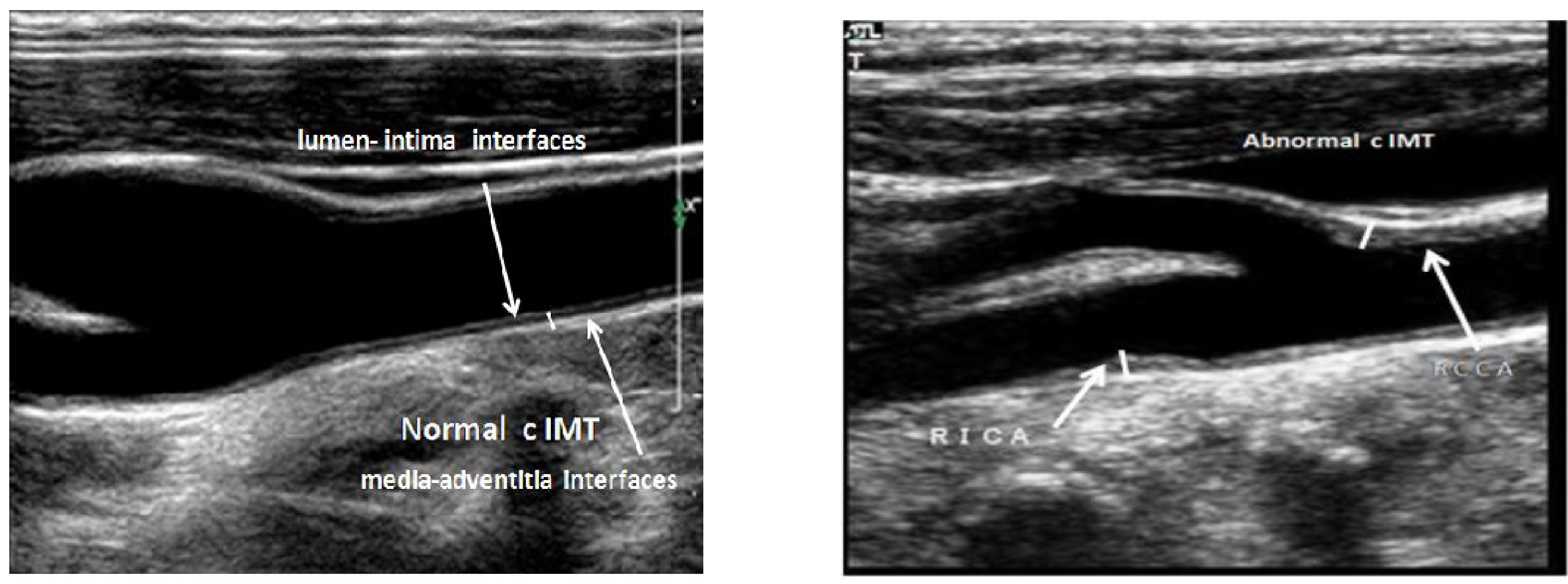

Figure 1. Normal cIMT measured as the distance between the lumen-intima and mediaadventitia interfaces (indicated by white flashes) of the far wall of common carotid artery (CCA). cIMT thickness is marked by a white short line.

Figure 2. Abnormal cIMT - thickening of intima and media of the vascular wall of CCA, the carotid bulb and the first $\mathrm{cm}$ of the internal carotid artery (ICA). cIMT thickness is marked by a white short line and the lumen-intima interface is indicated by a white flash.

\section{Results}

HS was correlated with NAFLD - FLI ( $\mathrm{p}$ 目 0.0001). Patients were divided into three groups: G1: FLI<30 ( $n=56)$; G3: FLI > $60(n=40), G 2$ : intermediate group $(n=28)$. The prevalence of NAFLD using FLI $\geq 60$ in DM patients was 40 patients (32\%). cIMT increased with FLI (G3 $=0.54 \pm 0.08$ versus $\mathrm{G} 1=0.40 \pm 0.08 \mathrm{~mm}, P<0.0001)$. FLI was associated with increased low-density lipoprotein cholesterol $(r=0.38)$, alanine aminotransferase $(r=0.55)$, BMI $(r=0.34)$, diastolic blood pressure $(r=$ $0.41)$ and reduced high-density lipoprotein cholesterol $(r=-0.52)$ and insulinemia ( $r=-0.30$, all $P<0.0001$ ). The correlations hold also in multivariate analysis after adjusting for age and gender. Behavioral variables (smoking, diet) and fasting plasma glucose, did not significantly differ between subjects with and without FLI.
Table 1 - Clinical and biochemical characteristics of diabetic patients grouped according to NAFLD

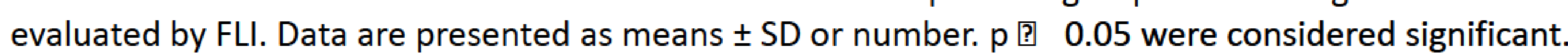

\begin{tabular}{|c|c|c|c|}
\hline Variable & $\begin{array}{l}\text { With NAFLD-FLI } \\
\text { mean } \pm S D \\
\text { (median) }\end{array}$ & $\begin{array}{l}\text { Without } \\
\text { NAFLD-FL } \\
\text { mean } \pm S D \\
\text { (median) }\end{array}$ & $\begin{array}{c}P \\
\text { (Mann Withney test) }\end{array}$ \\
\hline Age (years) & $55.7 \pm 8.9(56)$ & $58.7 \pm 8.5(58)$ & $p=0.005$ \\
\hline Diabetes duration (years) & $5.78 \pm 5.56(5.0)$ & $6.61 \pm 5.77(5.0)$ & $p=0.22$ \\
\hline $\mathrm{SBP}(\mathrm{mmHg})$ & $147.42 \pm 23.32(140)$ & $151.67 \pm 25.23(150)$ & $p=0.75$ \\
\hline DBP (mmHg) & $87.41 \pm 12.55(86)$ & $87.0 \pm 9.73(86)$ & $p=0.3$ \\
\hline $\mathrm{TC}(\mathrm{mmol} / \mathrm{l})$ & $5.21 \pm 1.7(5.1)$ & $4.67 \pm 1.25(4.68)$ & $p=0.006$ \\
\hline $\mathrm{HDLc}(\mathrm{mmol} / \mathrm{l})$ & $1.06 \pm 0.43(1.06)$ & $1.16 \pm 0.46(1.13)$ & $p=0.008$ \\
\hline $\operatorname{LDLc}(\mathrm{mmol} / \mathrm{l})$ & $2.79 \pm 1.62(2.99)$ & $2.80 \pm 1.18(2.94)$ & $p=0.82$ \\
\hline TGL/HDLC & $7.57 \pm 20.07(4.03)$ & $3.12 \pm 3.3(1.95)$ & $p 0.0001$ \\
\hline FPG (mmol/l) & $169.97 \pm 63.5(157)$ & $186.72 \pm 95.05(162.5)$ & $p=0.4$ \\
\hline HOMA-IR & $8.94 \pm 15.23(5.42)$ & $4.38 \pm 5.16(2.73)$ & $\mathrm{p}$ 国 0.0001 \\
\hline HbA1C & $10.53 \pm 40.8(7.9)$ & $8.2 \pm 1.87(7.9)$ & $p=0.99$ \\
\hline ASAT (units/I) & $29.43 \pm 18.8(24.5)$ & $21.43 \pm 9.23(20)$ & $\mathrm{p}$ Ð0.0001 \\
\hline ALAT (units/l) & $39.79 \pm 24.4(33)$ & $27.93 \pm 17.34$ (24.6) & $\mathrm{p} 0.0001$ \\
\hline VFA $\left(\mathrm{cm}^{2}\right)$ & $175.47 \pm 43.18(170.8)$ & $135.1 \pm 46.35(133.45)$ & $\mathrm{p} 0.0001$ \\
\hline $\operatorname{BFM}\left(\mathrm{cm}^{2}\right)$ & $36.36 \pm 14.07(34.2)$ & $25.35 \pm 12.78(23.1)$ & $\mathrm{p}$ 国 0.0001 \\
\hline PBF(\%) & $35.74 \pm 8.66$ (35.3) & $31.76 \pm 9.29$ (30.6) & $p=0.001$ \\
\hline
\end{tabular}

$B M I=$ body mass index, $S B P=$ systolic blood pressure, DBP $=$ diastolic blood pressure, $T C=$ total cholesterol, $H D L L=$ high density lipoprotein

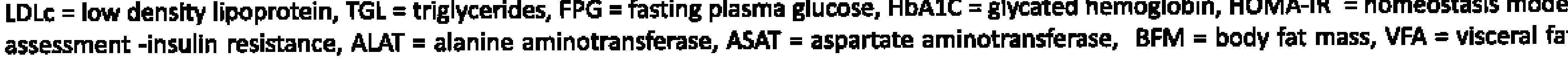
area, PFB (\%)= percent body fat.

Table 2. Multiple regression analysis. Model 1,2: age, sex, VFA, and ALAT are independently correlated with NAFLD-FLI

\begin{tabular}{|c|c|c|c|c|c|}
\hline \multirow[b]{2}{*}{ Model } & \multirow[b]{2}{*}{ Independent variable } & \multicolumn{2}{|c|}{ Unstandardized Coefficients } & \multirow{2}{*}{$\begin{array}{l}\text { Standardized } \\
\text { Coefficients } \\
\text { Beta }\end{array}$} & \multirow[b]{2}{*}{$\mathbf{P}$} \\
\hline & & B & Std Error & & \\
\hline \multirow{2}{*}{$\begin{array}{l}\text { Model } 1 \\
\mathrm{r}=0.387 \\
\mathrm{P} \text { 回 } 0.0001\end{array}$} & Age (years) & -0.007 & 0.002 & -0.161 & 0.001 \\
\hline & $\operatorname{VFA}\left(\mathrm{cm}^{2}\right)$ & 0.003 & 0.0001 & 0.362 & 0.0001 \\
\hline \multirow{3}{*}{$\begin{array}{l}\text { Model 2 } \\
r=0.409 \\
\text { PQ } 0.0001\end{array}$} & Age (years) & -0.006 & 0.002 & -0.138 & 0.004 \\
\hline & $\operatorname{VFA}\left(\mathrm{cm}^{2}\right)$ & 0.003 & 0.0001 & 0.341 & 0.0001 \\
\hline & Sex (male/female) & 0.117 & 0.041 & 0.137 & 0.005 \\
\hline \multirow{4}{*}{$\begin{array}{l}\text { Model } 3 \\
r=0.426 \\
\mathrm{PQ} 0.0001\end{array}$} & Age (years) & -0.005 & 0.002 & -0.119 & 0.013 \\
\hline & Sex (male/female) & 0.098 & 0.041 & 0.115 & 0.018 \\
\hline & VFA $\left(\mathrm{cm}^{2}\right)$ & 0.003 & 0.0001 & 0.336 & 0.0001 \\
\hline & ALAT (U/I) & 0.003 & 0.001 & 0.128 & 0.008 \\
\hline
\end{tabular}

Table 3. Multiple regression analysis. Model 1 and 2: age, HbA1C, HOMA-IR and VFA are independently correlated with cIMT

\begin{tabular}{|c|c|c|c|c|c|}
\hline \multirow[b]{2}{*}{ Model } & \multirow{2}{*}{$\begin{array}{l}\text { Independent } \\
\text { variable }\end{array}$} & \multicolumn{2}{|c|}{ Unstandardized Coefficients } & \multirow{2}{*}{$\begin{array}{l}\text { Standardized } \\
\text { Coefficients } \\
\text { Beta }\end{array}$} & \multirow[b]{2}{*}{$\mathbf{p}$} \\
\hline & & B & Standard Error & & \\
\hline \multirow{3}{*}{$\begin{array}{l}\text { Model } 1 \\
r=0.39 \\
P=0.002\end{array}$} & Age (years) & 0.009 & 0.004 & 0.22 & 0.023 \\
\hline & HbA1C (\%) & -0.001 & 0.0001 & -0.286 & 0.003 \\
\hline & HOMA-IR & 0.014 & 0.006 & 0.22 & 0.026 \\
\hline \multirow{4}{*}{$\begin{array}{l}\text { Model } 2 \\
r=0.45 \\
\text { P } 0.0001\end{array}$} & Age (years) & 0.006 & 0.002 & 0.27 & 0.001 \\
\hline & HbA1C(\%) & 0.033 & 0.011 & 0.24 & 0.004 \\
\hline & $\operatorname{VFA}\left(\mathrm{cm}^{2}\right)$ & 0.001 & 0.0001 & 0.27 & 0.001 \\
\hline & $\mathrm{SBP}(\mathrm{mmHg})$ & 0.001 & 0.001 & 0.15 & 0.074 \\
\hline
\end{tabular}

Conclusions:The results of our study are suggestive for the fact that, in T2DM patients, fatty liver is not a direct mediator of early carotid atherosclerosis. Our data indicate that visceral fat accumulation and glycated hemoglobin are determinant factors of cIMT suggesting that controlling abdominal obesity and hyperglicemia might reduce atherosclerotic disease risk in NAFLD-T2DM subjects. These findings support the hypothesis that the identification of NAFLD abdominal obese T2DM patients may help in CVD risk prediction, with important management implications.

\section{Bibliography}

Targher G, Bertolini L, Padovani R, et al. Relations between carotid artery thickness and liver histology in subjects with nonalcoholic fatty liver disease. Diabetes Care 2006: 29:1325-1330.

2. Petit JM, Guiu B, Terriat B et al. Nonalcoholic fatty liver is not associated with carotid intima-media thickness in type 2 diabetic patients. J Clin Endocrinol Metab 2009; 94: 4103-4106.

3. Bedogni G, Bellentani S, Miglioli L et al. The Fatty Liver Index : a simple and accurate predictor of hepatic steatosis in the genera population. BMC Gastroenterol. 2006 Nov 2;6:33.

Acknowledgement: This paper was published under the frame of European Social Found, Human Resources Development Operational Programme 2007-2013, project no. POSDRU/159/1.5/S/138776. 